\title{
Thermal-Piezoresistive Tuning of the Effective Quality Factor of a Micromechanical Resonator
}

\author{
James M. Lehto Miller, ${ }^{1, *}$ Haoshen Zhu, ${ }^{2, \dagger}$ David B. Heinz, ${ }^{1}$ Yunhan Chen, ${ }^{1}$ Ian B. Flader, ${ }^{1}$ \\ Dongsuk D. Shin, ${ }^{1}$ Joshua E.-Y. Lee, ${ }^{3}$ and Thomas W. Kenny ${ }^{1}$ \\ ${ }^{1}$ Department of Mechanical Engineering, Stanford University, Stanford, California 94305, USA \\ ${ }^{2}$ School of Electronic and Information Engineering, South China University of Technology, Guangzhou 510641, \\ China \\ ${ }^{3}$ State Key Laboratory of Millimeter Waves and the Department of Electronic Engineering, City University of \\ Hong Kong, Hong Kong
}

(Received 7 February 2018; revised manuscript received 6 August 2018; published 23 October 2018)

We study the thermal-piezoresistive pumping mechanism within silicon micromechanical resonators. We develop a multiphysics finite-element model to predict the effective quality factor tuning from pumping for arbitrary geometries and dopants. Our model reproduces the effective quality factor tuning direction and magnitude of our fabricated devices versus direct current as a function of dopant type, concentration, thermal actuator width, and device orientation. We show that, in contrast to degenerate parametric amplification, thermal pumping steepens the phase slope near resonance with an increasing effective quality factor.

DOI: 10.1103/PhysRevApplied.10.044055

\section{INTRODUCTION}

Microelectromechanical (MEM) and nanoelectromechanical (NEM) resonators underlie many sensors and oscillators used within academic research and technology, such as atomic-force microscopes [1], inertial sensors [2], timing references [3], and filters [4]. An important property of MEM resonators is the quality factor, $Q$, which is a measure of the energy dissipation in the mode. Effective quality factor $\left(Q_{\text {eff }}\right)$ tuning, such as external feedback control [5-8], parametric amplification [9-16], and optical pumping [17-20], is an active technique for feeding energy into or taking energy out of a mechanical mode, which artificially enhances or suppresses the measured quality factor of that mode. It is well known that $Q_{\text {eff }}$ tuning cannot modify the thermomechanical signal-to-noise ratio of a resonant sensor, because feedback identically modifies the resonator response to the signal and thermal noise $[21,22]$. $Q_{\text {eff }}$ suppression is helpful for improving the bandwidth $\mathrm{BW}=\omega_{n} / Q_{\text {eff }}$ of amplitude-modulated resonant sensors [23], and for ground state cooling of mechanical resonators

\footnotetext{
*jmlm@stanford.edu

†zhuhs@scut.edu.cn
}

Published by the American Physical Society under the terms of the Creative Commons Attribution 4.0 International license. Further distribution of this work must maintain attribution to the author(s) and the published article's title, journal citation, and DOI.
$[24,25] . Q_{\text {eff }}$ enhancement into the self-oscillation regime is useful as an oscillator topology [26,27].

Each $Q_{\text {eff }}$ tuning technique has trade-offs in terms of the feasibility of implementation, the power consumption, and the performance. External feedback control requires continuous measurement of the resonator thermal-noiseinduced motion and a means for feeding the phase-shifted signal back to drive the device. Degenerate parametric amplification requires the modulation of some parameter of the resonator, typically the spring constant, at twice its resonant frequency. Optical pumping requires the coupling of the mechanical mode to an optical or microwave cavity and the use of an external laser or microwave signal generator to pump the cavity at its red or blue sideband.

Thermal-piezoresistive pumping is a recently discovered technique for $Q_{\text {eff }}$ tuning of MEM/NEM resonators by converting energy from a direct current (dc) into alternating current (ac) motion [28]. Thermal pumping utilizes a thermoelectromechanical feedback mechanism within semiconductor structures with sufficient material piezoresistivity. The modification of $Q_{\text {eff }}$ is initiated by flowing a direct current through one or more beams in the structure, the longitudinal extension of which can excite the mode of interest [29]. The use of a simple direct current to tune $Q_{\text {eff }}$ is what distinguishes thermal pumping from the other methods. Figure 1 depicts how thermal pumping increases $Q_{\text {eff }}$ for a resonator with a negative piezoresistive coefficient (e.g., $n$-type doped silicon). Starting from the 


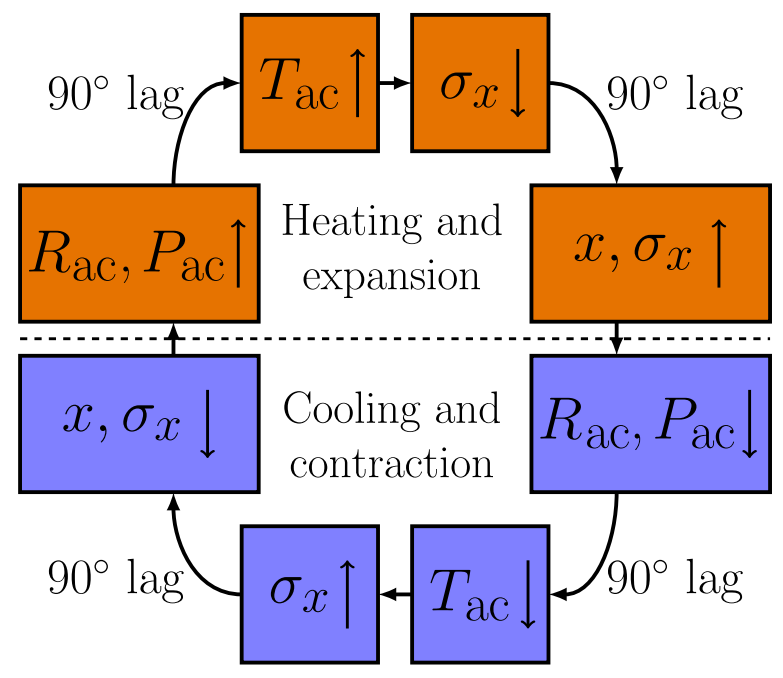

FIG. 1. The state diagram for an actuator beam with a negative longitudinal piezoresistive coefficient, depicting the changes in the resonator position $(x)$, actuator temperature $\left(T_{\mathrm{ac}}\right)$, actuator axial stress $\left(\sigma_{x}\right)$, actuator electrical resistance $\left(R_{\mathrm{ac}}\right)$, and Joule heating $\left(P_{\mathrm{ac}}\right)$ over one cycle. This feedback mechanism requires a constant current to flow through the actuator beam. For a negative (positive) piezoresistive coefficient, a tensile stress in the actuator beam causes the beam resistance to decrease (increase), which leads to resonator $Q_{\text {eff }}$ enhancement (suppression) with an increasing direct current.

left-hand side of Fig. 1, the resonator mass displaces to the left $(x<0)$, compressing the beam $\left(\sigma_{x}<0\right)$, which causes the electrical resistance to increase $\left(R_{\mathrm{ac}}>0\right)$ due to the negative piezoresistive coefficient, which in turn increases the Joule heating in the beam $\left(P_{\mathrm{ac}}>0\right)$. This increases the temperature in the beam $\left(T_{\mathrm{ac}}>0\right)$, which due to thermal expansion further increases the compressive stress. The mass moves back to its equilibrium position $(x=0)$ where its velocity is maximum. Due to inertia, the mass continues its motion $(x>0)$, which lowers the beam electrical resistance $\left(R_{\mathrm{ac}}<0\right)$, which reduces the Joule heating in the beam $\left(P_{\mathrm{ac}}<0\right)$ and lowers the temperature in the beam $\left(T_{\mathrm{ac}}<0\right)$ which, due to thermal contraction, further increases the tensile stress $\left(\sigma_{x}>0\right)$. The mass moves in the opposite direction, restarting the cycle. For sufficient current, the $Q_{\text {eff }}$ enhancement will lead to self-sustained oscillations of the resonator [30]. For a positive piezoresistive coefficient and a constant current, thermal pumping induces $Q_{\text {eff }}$ suppression.

Thermal pumping has been demonstrated in a variety of geometries fabricated from a variety of substrates, including silicon [31-34], group III-V semiconductors [35], and even complementary metal-oxide semiconductor (CMOS)-MEM devices [36]. It can be incorporated into conventional fabrication processes with minimal change to process flow and its implementation within sensors or oscillators does not require sophisticated external control electronics. The primary disadvantage of thermal pumping is the large power consumption associated with flowing a current through the resonator (several hundred microwatts to a few milliwatts is typical) and the associated change in resonator properties during heating. The large power consumption is an issue for thermal actuators in general [37] and can be reduced by shrinking resonators into the nanoscale [38].

Thermal pumping may enable $Q_{\text {eff }}$ tuning in handheld electronics, gigahertz frequency oscillators with submicrowatt power consumption, and in-cryostat signal generation for quantum computers. Thermal pumping can be integrated into the resonant sensors and oscillators used in handheld electronics. Unlike many of the other phase-independent $Q_{\text {eff }}$ tuning mechanisms, such as external feedback and optical pumping, thermal pumping does not require a bulky laboratory laser for position measurement or pumping. Because thermal pumping becomes more efficient at higher frequencies [39], it may eventually enable gigahertz frequency oscillators with submicrowatt power consumption. Quantum computing requires the coupling of microwave signals to quantum bits (qubits) that are cryogenically cooled into their quantum ground states [40-42], which requires bulky external microwave generators and cryostat cables that introduce parasitic reactance and added complexity. Thermal-piezoresistive selfoscillators may be helpful for integrating signal generators and qubits on the same chip, but cryogenic thermal pumping could require much higher dopant concentrations than previously considered to compensate for charge-carrier freezeout.

While a great deal of work has gone into characterizing and modeling the various dissipation mechanisms within MEM/NEM resonators, modeling of the various $Q_{\text {eff }}$ tuning techniques such as thermal pumping is still in its infancy [43]. Phan et al. developed a finite-element model to predict the threshold current in a lightly doped $n$-type flexural-mode thermal-piezoresistive resonator [31]. Rahafrooz and Pourkamali used a lumped element model to show that the self-oscillation threshold power scales quadratically with the resonator linear dimension [44]. Sundaram and Weinstein derived a lumped element model that predicts the threshold current in a variety of bulk-mode resonators as a function of material properties and resonator dimensions [29]. Miller et al. extended the model in [44] to predict the dependence of the threshold current on the ambient temperature [30].

An accurate and generalizable model of thermal pumping would be very helpful for designing new device architectures for different operating temperatures, reduced power consumption, and increased attainable selfoscillation frequency. Recent studies have shown that tailoring the dopant type and concentration of MEM/NEM resonators improves their frequency-temperature stability [45] and tunes their nonlinear behavior [46]. This paper demonstrates that doping concentration and residual 
heating strongly influences thermal pumping $Q_{\text {eff }}$ behavior with direct current and confirms this behavior with a generalizable model that accounts for the doping and temperature dependence of the piezoresistivity.

\section{DEVICE SETUP AND MODELING}

We fabricate our devices using a wafer-scale encapsulation process that produces stable, high-quality-factor resonators in a hermetic, vacuum-sealed environment (see Ref. [47]). Each device consists of a proof mass connected to two anchors via a wide "spring" beam and a narrow "engine" beam. The two anchors enable us to flow current through the resonator, tuning $Q_{\text {eff. }}$ The device resonates via a flexural mode that compresses and stretches the engine beam. Most of the electrical resistance of the device is in the engine beam, which is also the region that experiences the largest variations in stress during motion. The Joule heating from current flow is concentrated in the engine beam, inducing the coupled feedback cycle delineated in Fig. 1. The geometry considered has a beam length of $50 \mu \mathrm{m}$, a spring beam width of $12 \mu \mathrm{m}$, and an engine beam width of $3 \mu \mathrm{m}$. The predecessor to the device geometry used here was first developed for thermal actuation [48,49] and consisted of a polycrystalline silicon device with a wide beam directly connected to a narrow beam. Steeneken et al. later combined this thermal-actuation concept with a proof mass to create a single-crystal silicon resonator, the $Q_{\text {eff }}$ of which could be tuned using a direct current [28].

The micromechanical resonator and electrical test setup is depicted in Fig. 2. Our setup consists of a capacitive drive electrode, a capacitive sense electrode, a current source, and a piezoresistive readout. We apply a bias to the drive electrode and apply an ac voltage to actuate the device. We also apply a bias to the sense electrode and read out the motional current using a transimpedance amplifier. We piezoresistively read out the motion using a high-input-impedance amplifier. Given the relatively low

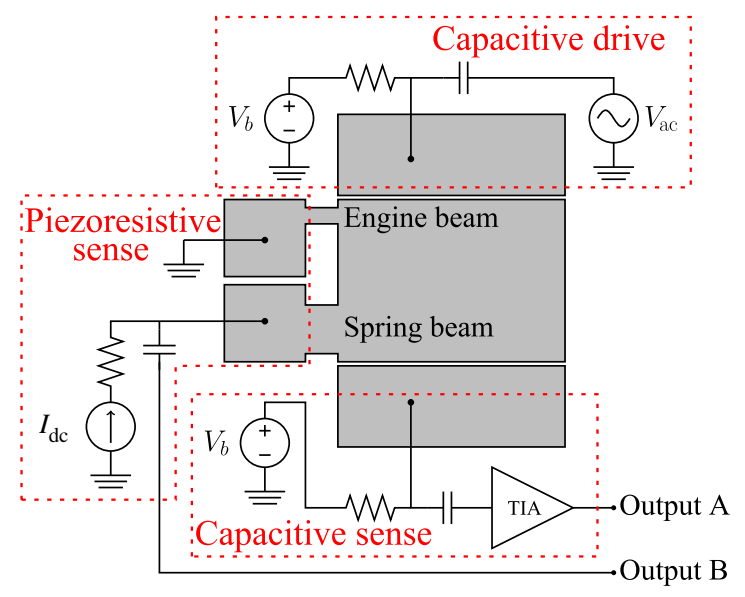

FIG. 2. The device with readout electronics.
TABLE I. The silicon wafers used for device fabrication. The first letter in the acronym specifies whether the dopant is an electron donor $(\mathrm{N})$ or an acceptor $(\mathrm{P})$. The second letter indicates the dopant element: phosphorus (P), antimony (S), or boron (B). The third letter gives the relative dopant concentration: moderate (M) or high $(\mathrm{H})$. The final indices give the wafer orientation.

\begin{tabular}{lcccc}
\hline \hline Wafer & Doping & $N\left(\mathrm{~cm}^{-3}\right)$ & $\rho_{0}(\Omega \mathrm{m})$ & Orientation \\
\hline NPH (100) & $n$-type (P) & $6.1 \times 10^{19}$ & $1.26 \times 10^{-5}$ & {$[100]$} \\
NSM (100) & $n$-type (Sb) & $1.3 \times 10^{18}$ & $1.90 \times 10^{-4}$ & {$[100]$} \\
NSM (111) & $n$-type (Sb) & $1.6 \times 10^{18}$ & $1.70 \times 10^{-4}$ & {$[110]$} \\
PBM (100) & $p$-type (B) & $6.4 \times 10^{18}$ & $1.22 \times 10^{-4}$ & {$[100]$} \\
PBH (100) & $p$-type (B) & $1.2 \times 10^{20}$ & $1.06 \times 10^{-5}$ & {$[100]$} \\
PBH (111) & $p$-type (B) & $2.5 \times 10^{19}$ & $4.25 \times 10^{-5}$ & {$[110]$} \\
\hline \hline
\end{tabular}

device electrical resistance and resonant frequency, electrical loading from the drive and sense circuitry can be neglected (see Ref. [50]).

We fabricate the resonators using silicon-on-insulator wafers of either $n$-type or $p$-type doping, with varying dopant concentration and wafer orientation. The devices are aligned parallel to the [100] direction in the (100) wafers and parallel to the [110] direction in the (111) wafers. The doping concentration $(N)$ varies from moderate to high doping. Table I summarizes the doping type, doping concentration, measured electrical resistivity $\left(\rho_{0}\right)$, and engine beam orientation of the resonators modeled and tested in this paper. The fabrication process is the same for all wafers, producing etch-hole-free encapsulated resonators, except for the heavily $n$-type doped devices, which have etch-holes.

To model the efficiency of the thermal-piezoresistive effect, we develop a fully coupled thermoelectromechanical model and implement it using COMSOL multiphysics [51]. Phan et al. reported a similar fully coupled model for predicting the self-oscillation current of lightly doped silicon thermal-piezoresistive resonators [31]. We use our model to predict the $Q_{\text {eff }}$ tuning behavior with current for $p$-type and $n$-type devices of varying dopings, orientations, and geometries. In our devices, the high doping concentration greatly degrades the piezoresistive coefficients but may be helpful for thermal pumping at low ambient temperatures. Moreover, the Joule heating that accompanies thermal pumping elevates the temperature of the device (mainly in the engine beam), which results in further deviation of our measurements from the model in Ref. [31]. We therefore incorporate the doping and temperature dependence of the piezoresistive coefficients into our model.

We first calculate the doping and temperature effect on piezoresistance based on Kanda's derivation [52]:

$$
\Pi_{i j}(N, T)=P(N, T) \Pi_{i j}(300 \mathrm{~K}),
$$

where $\Pi_{i j}(N, T)$ is the piezoresistive coefficient as a function of $N$ and temperature (T). For single-crystal silicon, $i j$ 
can be 11,12 , and $44 . \Pi_{i j}(300 \mathrm{~K})$ represents the measured piezoresistive coefficient at $300 \mathrm{~K}$ for lightly doped silicon. The piezoresistance factor $P(N, T)$ is given from Kanda as follows:

$$
P(N, T)=\frac{300}{T} F_{0}^{\prime}\left(\frac{E_{F}}{k_{B} T}\right) / F_{0}\left(\frac{E_{F}}{k_{B} T}\right),
$$

where $F_{0}\left(E_{F} / k_{B} T\right)$ is the Fermi-Dirac integral of order zero and $F_{0}^{\prime}\left(E_{F} / k_{B} T\right)$ is the derivative of $F_{0}\left(E_{F} / k_{B} T\right)$ with respect to $E_{F} / k_{B} T$ [52]. $E_{F}$ is the Fermi energy of the semiconductor, which is a function of $N$ as well as $T$. $k_{B}$ is the Boltzmann constant. Given the fixed $N$ (Table I), the temperature dependence of piezoresistance $\Pi_{i j}(T)$ can then be determined from Kanda [52]. Figure S2 in the Supplemental Material shows the piezoresistance factor as a function of temperature, $P(T)$, for the six different wafers used in this study [50]. We fit a 5th-degree polynomial to the nonlinear relation between temperature and piezoresistance factor for each of the six different wafers and then implement it into our COMSOL model.

The finite-element simulation of $Q_{\text {eff }}$ tuning is divided into two steps. In the first step, we perform a stationary study for a direct current $\left(I_{\mathrm{dc}}\right)$ to obtain the direct current density $\left(\mathbf{J}_{\mathrm{dc}}\right)$ distribution and elevated device temperature $\left(T_{\mathrm{dc}}\right)$ profile due to Joule heating [50,53]. This simulation (shown in Fig. S3 in the Supplemental Material) indicates that the highest $T_{\mathrm{dc}}$ occurs in the engine beam, which significantly decreases $\Pi_{i j}$ there. In the second step, we perform an eigenvalue study to solve the fully coupled partial differential equations and obtain the timevarying (ac) components [50,54-56]. We use the stationary results $\left(\mathbf{J}_{\mathrm{dc}}\right.$ and $\left.T_{\mathrm{dc}}\right)$ from the first step for the second step to determine the ac heating power density $\left(q_{\mathrm{ac}}\right)$ and spatial variation of $\Pi_{i j}\left(T_{\mathrm{dc}}\right)$ due to the localized dc Joule heating. The solutions of the displacement $\left(\mathbf{u}_{\mathrm{ac}}\right)$ and temperature $\left(T_{\mathrm{ac}}\right)$ for the coupled equations can be expressed as follows:

$$
\begin{aligned}
\mathbf{u}_{\mathrm{ac}}(\mathbf{r}, t) & =U_{\mathrm{ac}} \tilde{\boldsymbol{\Phi}}(\mathbf{r}) e^{\lambda t}, \\
T_{\mathrm{ac}}(\mathbf{r}, t) & =\Theta_{\mathrm{ac}} \tilde{T}(\mathbf{r}) e^{\lambda t},
\end{aligned}
$$

where $\mathbf{r}$ is the spatial coordinate vector, $t$ is time, $U_{\mathrm{ac}}$ and $\Theta_{\text {ac }}$ are the complex-valued modal magnitudes, $\tilde{T}(\mathbf{r})$ and $\tilde{\Phi}(\mathbf{r})$ are real-valued eigenmode functions, and $\lambda$ is the eigenvalue. The imaginary part of $\lambda$ is the resonant angular velocity and the real part indicates the damping coefficient. Using an experimentally measured $Q$ and the computed eigenvalue $\lambda$, we calculate the effective quality factor for a given direct current using

$$
\frac{1}{Q_{\mathrm{eff}}\left(I_{\mathrm{dc}}\right)}=\frac{1}{Q\left(I_{\mathrm{dc}}=0\right)}+\frac{1}{Q_{\mathrm{TPP}}\left(I_{\mathrm{dc}}\right)},
$$

where $Q_{\mathrm{TPP}}=\operatorname{Im}(\lambda) /(2 \operatorname{Re}(\lambda))$ indicates the quality factor induced by thermal-piezoresistive pumping. $Q$ is the mechanical quality factor, which is obtained for $I_{\mathrm{dc}}=0$ $\mathrm{mA}$. When $T_{\mathrm{ac}}$ in the engine beam is positive, the mechanical displacements are out of phase for the $n$-type and $p$-type devices due to their opposite longitudinal piezoresistive coefficients. For example, $\lambda$ for an $n$-type doped device yields a negative $Q_{\mathrm{TPP}}$, indicating that the device is gaining energy due to the thermal-piezoresistive effect. For a $p$-type device, the positive $Q_{\text {TPP }}$ suggests energy loss, without contributing any fluctuations characteristic of intrinsic losses [57].

The force exerted by the thermal actuator during pumping is $F_{t}=\beta I_{\mathrm{dc}}^{2} k_{l} x$, where $k_{l}$ is the lumped spring constant of the mode and $\beta$ is a complex-valued coefficient that indicates the efficiency of thermal pumping [28]. $\operatorname{Re}\{\beta\}$ characterizes the change in effective stiffness and $\operatorname{Im}\{\beta\}$ characterizes the change in effective damping with current. The effective quality factor is related to $\operatorname{Im}\{\beta\}$ and the $Q$ as follows:

$$
Q_{\mathrm{eff}}=\frac{Q}{1-\operatorname{Im}\{\beta\} Q I_{\mathrm{dc}}^{2}} .
$$

We can compute $\operatorname{Im}\{\beta\}$ from $\lambda$ at a given current using

$$
\operatorname{Im}\{\beta\}=-\frac{2 \operatorname{Re}\{\lambda\}}{\operatorname{Im}\{\lambda\} I_{\mathrm{dc}}^{2}} .
$$

In the absence of electrical loading, $\beta$ only depends on the device geometry and material properties. For small currents, $\beta$ is constant, $\operatorname{so} \operatorname{Im}\{\beta\}$ is useful for comparing the thermal pumping efficiency of different geometries and materials. $\operatorname{Im}\{\beta\}$ is positive for $Q_{\text {eff }}$ enhancement and negative for $Q_{\text {eff }}$ suppression. $|\operatorname{Im}\{\beta\}|$ should be made as large as possible to optimize thermal pumping. Our model predicts $\operatorname{Im}\{\beta\}$ from first principles, which is very helpful for optimizing thermal pumping prior to device fabrication.

\section{EXPERIMENTS}

We use the ring-down method and the 3-decibel (3-dB) method to measure $Q_{\text {eff }}$ of our thermal-piezoresistive resonators as a function of $I_{\mathrm{dc}}$. The ring-down technique measures $Q_{\text {eff }}$ in the time domain using the rate of resonator-amplitude decay after the driving force is switched off. The 3-dB method measures the quality factor in the frequency domain by applying a driving force of increasing frequency, obtaining the amplitude versus frequency curve, and measuring the width of the resonance peak. For low-frequency and high- $Q$ devices (e.g., our resonators), the ring-down technique is a more accurate measure of $Q_{\text {eff }}$ over the conventional 3-dB method, as it is usually difficult to achieve ultrafine frequency resolution to accurately determine the 3-dB bandwidth. Additionally, resonant-frequency fluctuations (especially for large currents) during the frequency sweep can result in an inaccurate bandwidth measurement. The ring-down method also eliminates the electronic loading effect on $Q_{\text {eff }}$. 

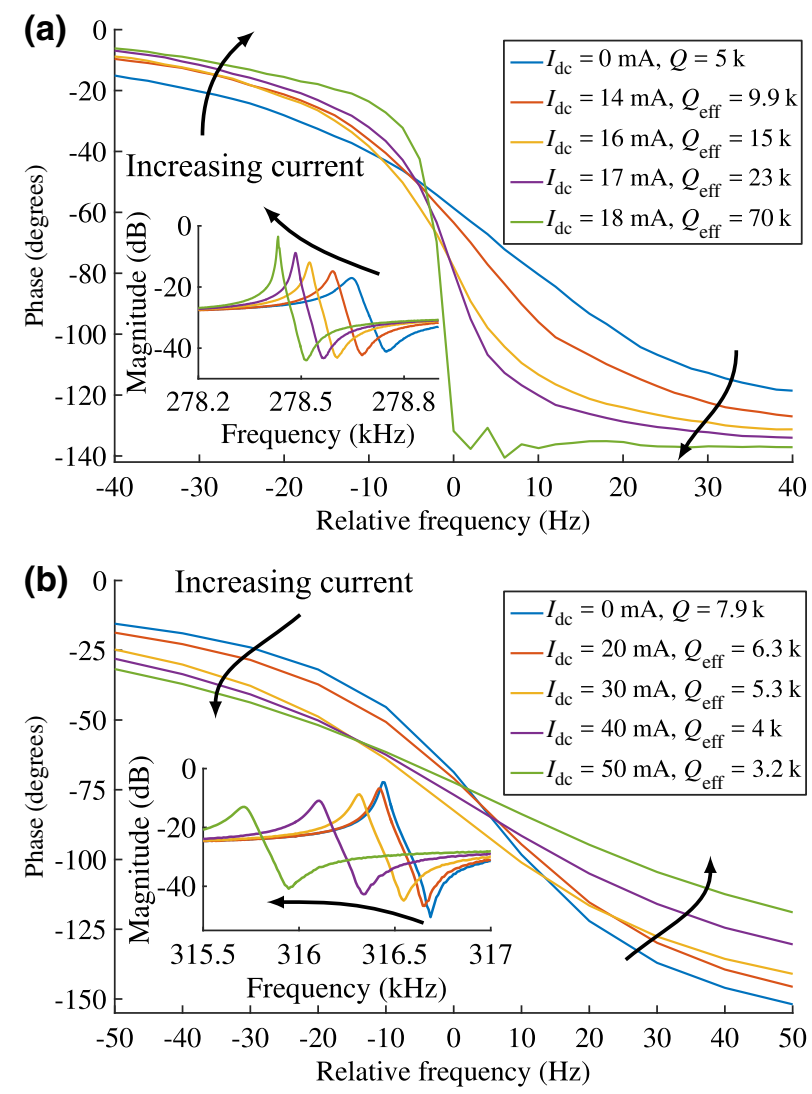

FIG. 3. (a) The measured $S_{21}$ phase and magnitude (inset) versus frequency of an $n$-type doped resonator for increasing current, showing $Q_{\text {eff }}$ enhancement. (b) The same measurement for a $p$-type doped resonator, showing $Q_{\text {eff }}$ suppression.

We first measure the transmission magnitude and phase for increasing currents through our resonators using a network analyzer. Figure 3(a) shows that $Q_{\text {eff }}$ is enhanced by more than tenfold for our moderately $n$-type doped [100] device with increasing current, as measured using the $3-\mathrm{dB}$ method. With increasing $Q_{\text {eff }}$ enhancement, the transmission becomes more sharply peaked and the phase slope steepens. This suggests that thermal-piezoresistive pumping is a phase-independent $Q_{\text {eff }}$ tuning mechanism, analogous to external feedback and optical pumping, in contrast to degenerate parametric amplification, where an increasing $Q_{\text {eff }}$ corresponds to a more sharply peaked magnitude but a less steep phase slope [58]. The contradictory phase-slope behavior is one of the differences between phase-independent and phase-dependent $Q_{\text {eff }}$ tuning mechanisms, and can be predicted using perturbation theory [59]. Figure $3(\mathrm{~b})$ shows that during $Q_{\text {eff }}$ suppression of a heavily $p$-type doped [110] device, the transmission amplitude broadens and the phase slope becomes less steep.

We next characterize $Q_{\text {eff }}$ via the ring-down method at each $I_{\mathrm{dc}}$ using both the capacitive and piezoresistive readouts (for a discussion of the trade-offs of these two different motion transduction techniques, see Ref. [50]). After recording the vibration decay at a given $I_{\mathrm{dc}}$, we measure the quality factor at several segments along the trajectory. The extracted $Q_{\text {eff }}$ values are clustered together for a given current, which suggests that there is negligible nonlinear damping at the starting drive amplitude $[60,61]$. Since we simultaneously measure each ring-down capacitively and piezoresistively, we use both time series to
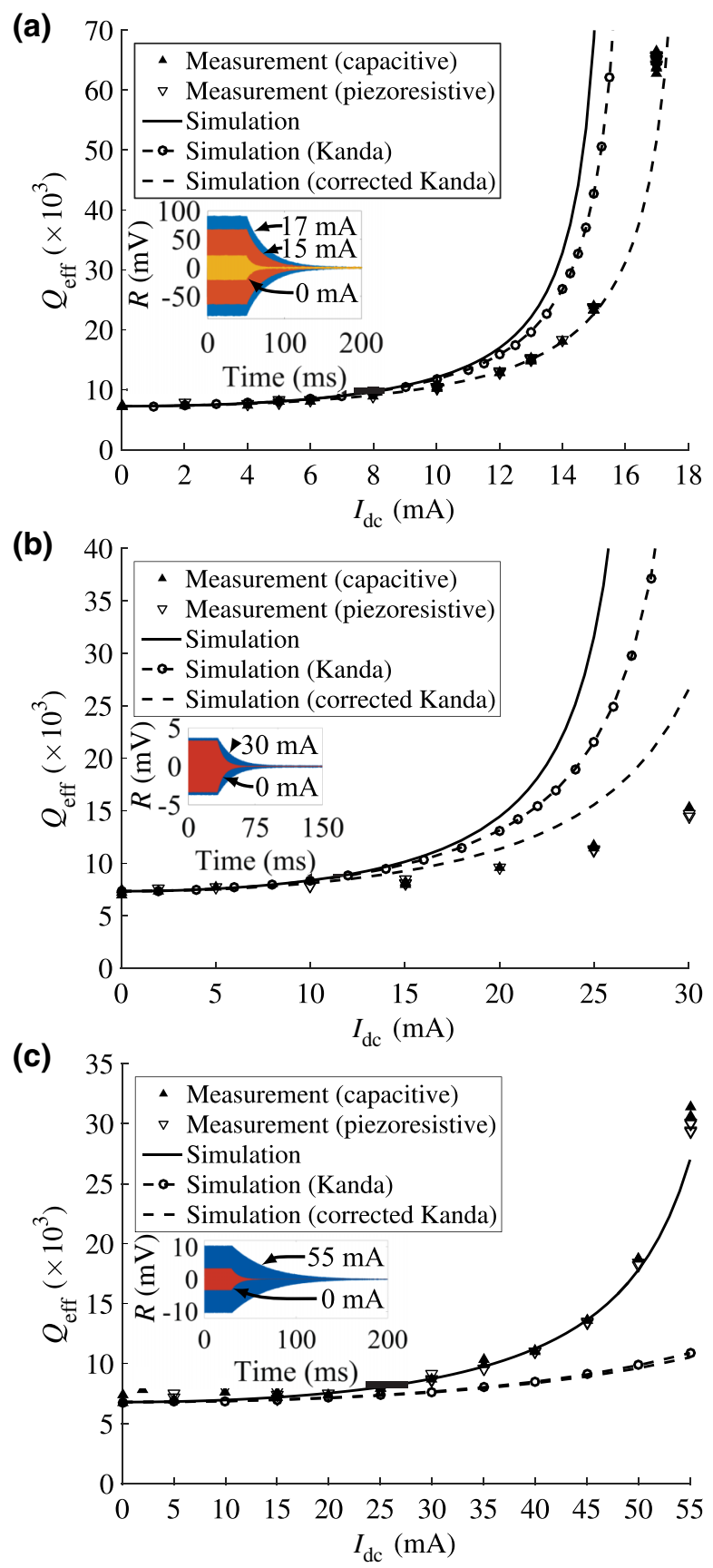

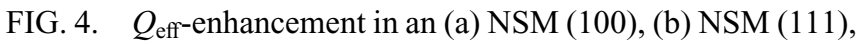
and (c) NPH (100) device using the ring-down technique. Insets: the measured ring-down amplitude $(R)$ versus time at various direct currents $\left(I_{\mathrm{dc}}\right)$ using the capacitive readout. 

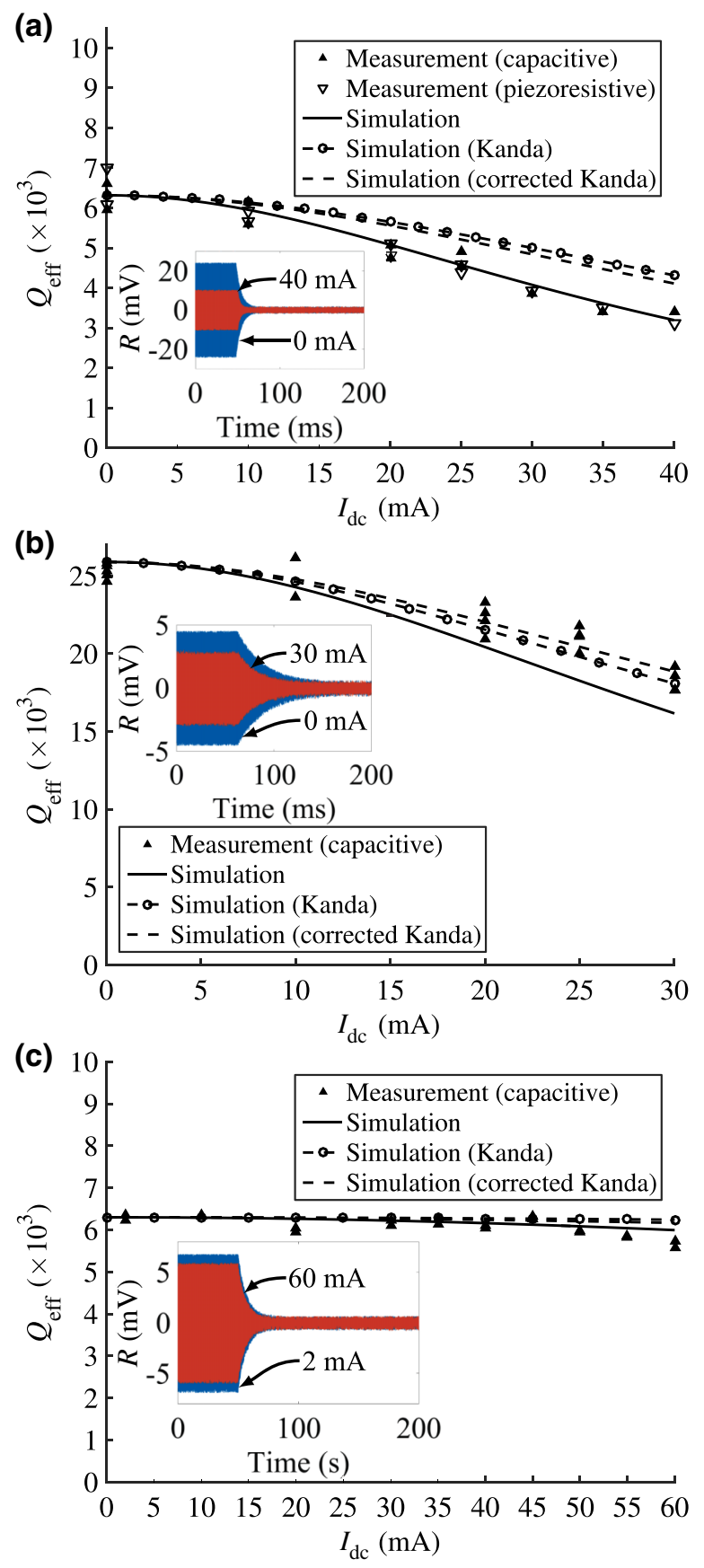

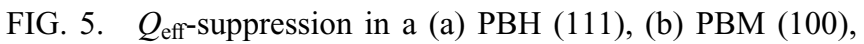
and (c) $\mathrm{PBH}$ (100) device using the ring-down technique. Insets: the measured ring-down amplitude $(R)$ versus time at various direct currents $\left(I_{\mathrm{dc}}\right)$ using the capacitive readout.

compute $Q_{\text {eff }}$ for each $I_{\mathrm{dc}}$. The estimates for $Q_{\text {eff }}$ generally closely agree between the two readout techniques, which corroborates the accuracy of the measurements. All measurements are performed in a temperature controlled chamber at $298 \mathrm{~K}$.

We plot the $Q_{\text {eff }}$ tuning for the $n$-type devices in Fig. 4 and for the $p$-type devices in Fig. 5, along with the

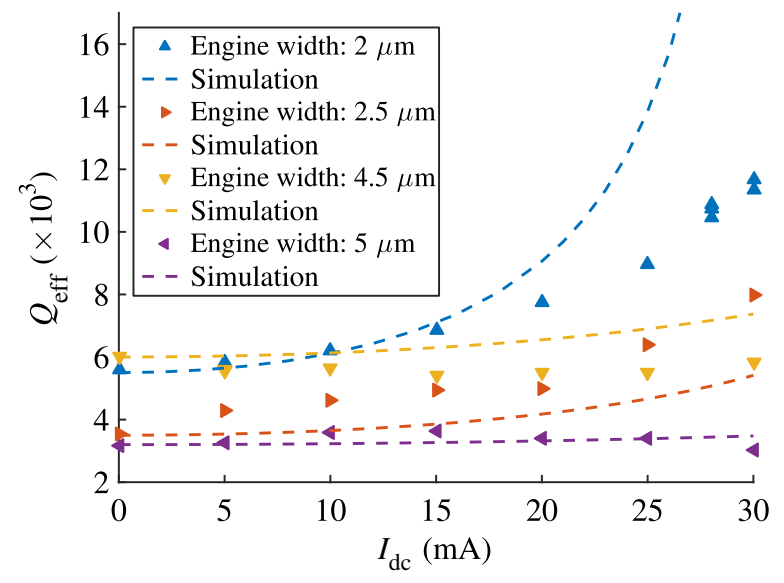

FIG. 6. $Q_{\text {eff-enhancement in the wide-spring-beam NSM (100) }}$ devices as a function of engine beam width, using the capacitive readout. With increasing engine-beam width, the $Q_{\text {eff- }}$ enhancement at a given direct current $\left(I_{\mathrm{dc}}\right)$ is reduced.

predictions from our finite-element model. The solid line indicates the simulation without accounting for the doping and temperature dependence of the piezoresistance. The dashed-dotted and dashed lines represent the simulations accounting for the doping and temperature dependence of the piezoresistance by adopting the Kanda model and a corrected Kanda model, respectively (for a detailed discussion, see Sec. IV). Of the $n$-type doped wafers, the moderately $n$-type doped device in the [100] orientation shows the most significant $Q_{\text {eff }}$ enhancement with direct current and thus the best thermal-piezoresistive efficiency. This is largely due to the higher longitudinal piezoresistance for the engine beam aligned in the [100] orientation with relatively low dopant concentration, while either a higher concentration or a different orientation (e.g., [110]) will reduce the longitudinal piezoresistance. The longitudinal piezoresistance for a (100) $n$-type doped wafer is maximized in the [100] orientation. Of the $p$-type doped wafers, the device on the heavily $p$-type doped (111) wafer shows the most significant $Q_{\text {eff }}$ suppression with current and thus the best thermal-piezoresistive efficiency. In this case, the [110] device orientation on the wafer corresponds to the largest possible piezoresistive coefficient and hence more efficient thermal pumping, while the [100] orientation corresponds to a nearly zero piezoresistive coefficient. The thermal-piezoresistive efficiency can be further improved by using [110] devices with a lighter $p$-type doping.

We also study the influence of engine beam width on thermal pumping in Fig. 6 . We choose the moderately $n$ type doped (100) wafer because the thermal-piezoresistive effect is strongest for this doping and orientation. We use devices with a beam length of $20 \mu \mathrm{m}$, a spring beam width of $60 \mu \mathrm{m}$, and a variety of engine beam widths from 2 to $5 \mu \mathrm{m}$. The proof-mass dimensions are the same 
as for the device tested in Fig. 4(a). We observe that thermal pumping becomes progressively less efficient as we increase the engine-beam width, which agrees with our model. The wider spring beam corresponds to a higher resonant frequency (about $500 \mathrm{kHz}$ ) and thus a larger actuation force is required to achieve the same displacement. A wider engine beam also corresponds to a lower current density for a given direct current, which corresponds to a smaller temperature variation in the engine beam for a given displacement. A shorter engine beam corresponds to a smaller change in actuator resistance for a given piezoresistivity and stress amplitude. All of these factors correspond to a lower thermal-piezoresistive efficiency than the geometry tested in Fig. 4(a).

\section{DISCUSSION AND CONCLUSIONS}

We observe that after incorporation of the theoretical Kanda model to account for the doping and temperature dependence of the piezoresistance (the dashed-dotted lines in Figs. 4 and 5), our simulations still deviate from the measurement results. In particular, the simulations overestimate the variation of $Q_{\text {eff }}$ at higher $I_{\mathrm{dc}}$ for moderately doped devices while underestimating the $Q_{\text {eff }}$ variation for highly doped devices. We initially attributed this discrepancy to the temperature dependence of the damping (i.e., thermoelastic dissipation [62]) or other material properties (i.e., electrical resistivity), but follow-up finite-element simulations have indicated that thermoelastic dissipation and resistivity only have a minor effect on thermal pumping (see Ref. [50]). We conclude that a more likely cause is the inaccuracy of the Kanda model in predicting the doping effect of piezoresistivity. Previous experimental studies have found that the measured piezoresistivity starts to deviate from the Kanda model at an impurity concentration as low as $10^{17} \mathrm{~cm}^{-3}$ for $p$-type doping [63] and $10^{16} \mathrm{~cm}^{-3}$ for $n$-type doping [64]. For the moderately doped samples, the actual piezoresistivity should be noticeably lower than the value predicted by the Kanda model. By correcting the Kanda model predictions of the doping effect on the piezoresistivity with an empirical factor based on the experimental data in $[63,64]$, the simulation (the dashed lines in Figs. 4 and 5) more closely agrees with our measurements of the moderately doped devices. However, the simulations with the corrected Kanda model underestimate the thermal-piezoresistive efficiency for the highly doped devices, which warrants further investigation. One possible cause for the discrepancy could be the variation in the fabricated geometry from the simulated geometry. As shown in Fig. 6, the thermal-piezoresistive efficiency is sensitive to the engine beam width and our simulations suggest that an overetch of $500 \mathrm{~nm}$ in the engine beam of the heavily doped devices would yield complete agreement with the experiments. Additionally, the fabrication process of the heavily $n$-type doped device is different from
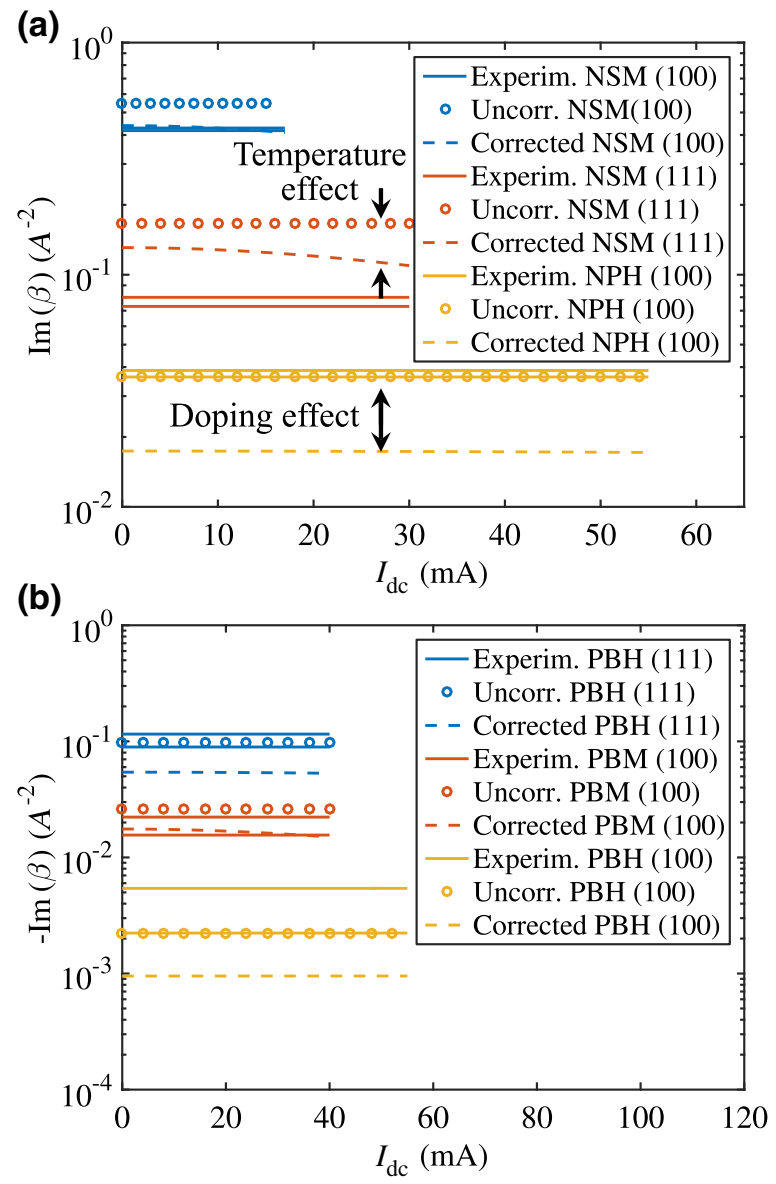

FIG. 7. The experimental and simulated thermal-piezoresistive efficiency factor $(\operatorname{Im}\{\beta\})$ versus direct current $\left(I_{\mathrm{dc}}\right)$ for the (a) NSM (100), NPH (100), and NSM (111) devices, and the (b) PBH (111), PBM (100), and PBH (100) devices. The upper and lower solid lines for each device correspond to the $95 \%$ confidence intervals for $\operatorname{Im}\{\beta\}$. We additionally plot uncorrected simulations and simulations that use the corrected Kanda model for the doping and temperature dependence of the piezoresistivity.

others and has etch holes perforated throughout the proof mass. In future device fabrication runs, the critical dimensions of all of the engine beams will be measured prior to the encapsulation process and the heavily $n$-type doped devices will be refabricated using the same process as for the other devices. Moreover, to validate the doping effect on piezoresistivity for our devices, test structures will be included into each wafer that enable us to experimentally extract the doping.

The $\beta$ parameter that we discuss in Sec. II is the most important metric for thermal-piezoresistive performance. In Fig. 7, we compare the simulated value of $\operatorname{Im}\{\beta\}$ to the experimentally extracted value for our devices. We observe significant degradation of the nominal $\operatorname{Im}\{\beta\}$ in the heavily doped $n$-type and $p$-type wafers. In the moderately $n$-type [110] and moderately $p$-type [100] devices, the nominal $\operatorname{Im}\{\beta\}$ degrades at large currents due to the degradation of 
the piezoresistive coefficients with increased device temperature. We extract the experimental $\operatorname{Im}\{\beta\}$ value for each device by fitting Eq. (6) to the measured $Q_{\text {eff }}$ values (shown in Fig. S5 in the Supplemental Material). Since the temperature dependence of $\operatorname{Im}\{\beta\}$ is still not significant at the applied currents, a good fit between the experiment and the model is possible for constant $|\operatorname{Im}\{\beta\}|$. The experimental $\operatorname{Im}\{\beta\}$ values agree fairly well with the simulated values, particularly for the moderately doped devices, and decreases by more than an order of magnitude for the same geometry when the doping is increased from moderate to heavy doping.

In this paper, we demonstrate that thermal-piezoresistive $Q_{\text {eff }}$ tuning can be predicted using a coupled finite-element model. We experimentally and theoretically show that $|\operatorname{Im}\{\beta\}|$ can vary by nearly 2 orders of magnitude for the same geometry by changing the wafer orientation, dopant type, and doping concentration. There is room to improve $\operatorname{Im}\{\beta\}$ by several orders of magnitude from the values we report here and thus dramatically improve the thermal-piezoresistive efficiency [28]. To minimize the power consumption of thermal pumping, the geometry, doping, and device orientation should be optimized. For $n$-type doped devices, the [100] engine beam alignment is optimum, while for $p$-type doped devices, the [110] engine beam alignment is optimum in-plane. For a given piezoresistivity, increasing the engine beam length will increase the piezoresistance and hence the thermalpiezoresistive effect. To increase the current density in the engine beam, the cross-section area of the engine beam should be reduced, either by reducing the beam width or by reducing the device layer thickness. To maximize the piezoresistive coefficients, the doping concentration should be well below $10^{19} \mathrm{~cm}^{-3}$ for both the $n$-type and $p$ type devices. The model that we discuss here will be helpful for predicting $\operatorname{Im}\{\beta\}$, and thus thermal-piezoresistive efficiency, during device design.

\section{ACKNOWLEDGMENTS}

Fabrication was performed in the nano@Stanford laboratories, which are supported by the National Science Foundation as part of the National Nanotechnology Coordinated Infrastructure under award No. ECCS-1542152, with support from the Defense Advanced Research Projects Agency Precise Robust Inertial Guidance for Munitions (PRIGM) Program, managed by Dr. Robert Lutwak. J.M.L.M. is supported by the National Defense Science and Engineering Graduate (NDSEG) Fellowship and the E.K. Potter Stanford Graduate Fellowship. J.M.L.M. is grateful to Alireza Ramezany and Wentao Jiang for helpful discussions, and to Rishi N. Patel for proofreading the manuscript. H.Z. is supported in part by the Fundamental Research Funds for the Central Universities under Grant No. 2018MS17 and in part by the Guangdong Innovative and Entrepreneurial Research Team Program under Grant No. 2017ZT07X032.

[1] F. J. Giessibl, Advances in atomic force microscopy, Rev. Mod. Phys. 75, 949 (2003).

[2] R. Bogue, MEMS sensors: Past, present and future, Sens. Rev. 27, 7 (2007).

[3] www.sitime.com, SiTime, Inc., Sunnyvale, CA (2018).

[4] C. T. Nguyen, MEMS technology for timing and frequency control, IEEE Trans. Ultrason. Ferroelectr. Freq. Control 54, 251 (2007).

[5] J. Mertz, O. Marti, and J. Mlynek, Regulation of a microcantilever response by force feedback, Appl. Phys. Lett. 62, 2344 (1993).

[6] C. H. Liu and T. W. Kenny, A high-precision, widebandwidth micromachined tunneling accelerometer, J. Microelectromech. Syst. 10, 425 (2001).

[7] D. Kleckner and D. Bouwmeester, Sub-kelvin optical cooling of a micromechanical resonator, Nature 444, 75 (2006).

[8] D. Wilson, V. Sudhir, N. Piro, R. Schilling, A. Ghadimi, and T. J. Kippenberg, Measurement-based control of a mechanical oscillator at its thermal decoherence rate, Nature 524, 325 (2015).

[9] D. Rugar and P. Grütter, Mechanical Parametric Amplification and Thermomechanical Noise Squeezing, Phys. Rev. Lett. 67, 699 (1991).

[10] J. F. Rhoads, N. J. Miller, S. W. Shaw, and B. F. Feeny, Mechanical domain parametric amplification, J. Vibr. Acoust. 130, 061006 (2008).

[11] H. Chan, M. I. Dykman, and C. Stambaugh, Paths of Fluctuation Induced Switching, Phys. Rev. Lett. 100, 130602 (2008)

[12] I. Mahboob and H. Yamaguchi, Bit storage and bit flip operations in an electromechanical oscillator, Nat. Nanotechnol. 3, 275 (2008).

[13] J. Suh, M. D. LaHaye, P. M. Echternach, K. C. Schwab, and M. L. Roukes, Parametric amplification and back-action noise squeezing by a qubit-coupled nanoresonator, Nano Lett. 10, 3990 (2010).

[14] M. J. Thompson and D. A. Horsley, Parametrically amplified $z$-axis Lorentz force magnetometer, J. Microelectromech. Syst. 20, 702 (2011).

[15] R. Karabalin, R. Lifshitz, M. Cross, M. Matheny, S. Masmanidis, and M. Roukes, Signal Amplification by Sensitive Control of Bifurcation Topology, Phys. Rev. Lett. 106, 094102 (2011).

[16] C. H. Ahn, S. Nitzan, E. J. Ng, V. A. Hong, Y. Yang, T. Kimbrell, D. A. Horsley, and T. W. Kenny, Encapsulated high frequency $(235 \mathrm{kHz})$, high- $Q(100 \mathrm{k})$ disk resonator gyroscope with electrostatic parametric pump, Appl. Phys. Lett. 105, 243504 (2014).

[17] T. J. Kippenberg and K. J. Vahala, Cavity optomechanics: Back-action at the mesoscale, Science 321, 1172 (2008).

[18] C. H. Metzger and K. Karrai, Cavity cooling of a microlever, Nature 432, 1002 (2004).

[19] A. G. Krause, M. Winger, T. D. Blasius, Q. Lin, and O. Painter, A high-resolution microchip optomechanical accelerometer, Nat. Photonics 6, 768 (2012). 
[20] G. Bahl, M. Tomes, F. Marquardt, and T. Carmon, Observation of spontaneous Brillouin cooling, Nat. Phys. 8, 203 (2012).

[21] J. Tamayo, Study of the noise of micromechanical oscillators under quality factor enhancement via driving force control, J. Appl. Phys. 97, 044903 (2005).

[22] A. Vinante, M. Bonaldi, F. Marin, and J. Zendri, Dissipative feedback does not improve the optimal resolution of incoherent force detection, Nat. Nanotechnol. 8, 470 (2013).

[23] K. J. Bruland, J. L. Garbini, W. M. Dougherty, and J. A. Sidles, Optimal control of ultrasoft cantilevers for force microscopy, J. Appl. Phys. 83, 3972 (1998).

[24] J. Teufel, T. Donner, D. Li, J. Harlow, M. Allman, K. Cicak, A. Sirois, J. D. Whittaker, K. Lehnert, and R. W. Simmonds, Sideband cooling of micromechanical motion to the quantum ground state, Nature 475, 359 (2011).

[25] A. H. Safavi-Naeini, J. Chan, J. T. Hill, T. P. M. Alegre, A. Krause, and O. Painter, Observation of Quantum Motion of a Nanomechanical Resonator, Phys. Rev. Lett. 108, 033602 (2012).

[26] C. Chen, D. H. Zanette, J. R. Guest, D. A. Czaplewski, and D. López, Self-Sustained Micromechanical Oscillator with Linear Feedback, Phys. Rev. Lett. 117, 017203 (2016).

[27] L. G. Villanueva, R. B. Karabalin, M. H. Matheny, E. Kenig, M. C. Cross, and M. L. Roukes, A nanoscale parametric feedback oscillator, Nano Lett. 11, 5054 (2011).

[28] P. G. Steeneken, K. Le Phan, M. J. Goossens, G. E. J. Koops, G. J. A. M. Brom, C. van der Avoort, and J. T. M. van Beek, Piezoresistive heat engine and refrigerator, Nat. Phys. 7, 354 (2011).

[29] S. Sundaram and D. Weinstein, Bulk mode piezoresistive thermal oscillators: Time constants and scaling, IEEE Trans. Ultrason. Ferroelectr. Freq. Control 62, 1554 (2015).

[30] J. M. L. Miller, D. B. Heinz, I. B. Flader, Y. Chen, D. D. Shin, and T. W. Kenny, in Proceedings IEEE Transducers, Kaohsiung, Taiwan (IEEE, New York, 2017), p. 1907.

[31] K. L. Phan, P. G. Steeneken, M. J. Goossens, G. E. J. Koops, G. J. A. M. Verheijden, and J. van Beek, Spontaneous mechanical oscillation of a de driven single crystal, arXiv:0904.3748 (2009).

[32] H. Zhu, C. Tu, and J. E.-Y. Lee, in Proceedings IEEE MEMS, Taipei, Taiwan (IEEE, New York, 2013), p. 757.

[33] G. Lehée, F. Souchon, J.-C. Riou, A. Bosseboeuf, and G. Jourdan, in Proceedings IEEE MEMS, Shanghai, China (IEEE, New York, 2016), p. 99.

[34] A. Ramezany, M. Mahdavi, and S. Pourkamali, Nanoelectromechanical resonant narrow-band amplifiers, Microsyst. Nanoeng. 2, 16004 (2016).

[35] A. Ansari and M. Rais-Zadeh, in Proceedings IEEE MEMS, Shanghai, China (IEEE, New York, 2016), p. 123.

[36] T.-Y. Liu, C.-C. Chu, M.-H. Li, and C.-Y. Liu, in Proceedings IEEE Transducers, Kaohsiung, Taiwan (IEEE, New York, 2017), p. 452.

[37] N. C. Tien, Silicon micromachined thermal sensors and actuators, Microscale Thermophys. Eng. 1, 275 (1997).

[38] A. Ramezany and S. Pourkamali, Ultrahigh frequency nanomechanical piezoresistive amplifiers for direct channel-selective receiver front-ends, Nano Lett. 18, 2551 (2018).
[39] A. Rahafrooz and S. Pourkamali, High-frequency thermally actuated electromechanical resonators with piezoresistive readout, IEEE Trans. Electron Devices 58, 1205 (2011).

[40] Y. Nakamura, Y. A. Pashkin, and J. Tsai, Coherent control of macroscopic quantum states in a single-Cooper-pair box, Nature 398, 786 (1999).

[41] D. P. DiVincenzo, The physical implementation of quantum computation, Fort. Phys. 48, 771 (2000).

[42] T. D. Ladd, F. Jelezko, R. Laflamme, Y. Nakamura, C. Monroe, and J. L. O'Brien, Quantum computers, Nature 464, 45 (2010).

[43] M. Imboden and P. Mohanty, Dissipation in nanoelectromechanical systems, Phys. Rep. 534, 89 (2014).

[44] A. Rahafrooz and S. Pourkamali, Thermal-piezoresistive energy pumps in micromechanical resonant structures, IEEE Trans. Electron Devices 59, 3587 (2012).

[45] A. Jaakkola, M. Prunnila, T. Pensala, J. Dekker, and P. Pekko, Design rules for temperature compensated degenerately $n$-type-doped silicon MEMS resonators, J. Microelectromech. Syst. 24, 1832 (2015).

[46] Y. Yang, E. J. Ng, P. M. Polunin, Y. Chen, I. B. Flader, S. W. Shaw, M. I. Dykman, and T. W. Kenny, Nonlinearity of degenerately doped bulk-mode silicon MEMS resonators, J. Microelectromech. Syst. 25, 859 (2016).

[47] Y. Yang, E. J. Ng, Y. Chen, I. B. Flader, and T. W. Kenny, A unified epi-seal process for fabrication of highstability microelectromechanical devices, J. Microelectromech. Syst. 25, 489 (2016).

[48] J. H. Comtois and V. M. Bright, Applications for surfacemicromachined polysilicon thermal actuators and arrays, Sens. Actuators A 58, 19 (1997).

[49] J. T. Butler, V. M. Bright, and W. D. Cowan, Average power control and positioning of polysilicon thermal actuators, Sens. Actuators A 72, 88 (1999).

[50] See the Supplemental Material at http://link.aps.org/supple mental/10.1103/PhysRevApplied.10.044055 for additional details about device modeling and measurements.

[51] COMSOL multiphysics, Burlington, MA (2015).

[52] Y. Kanda, A graphical representation of the piezoresistance coefficients in silicon, IEEE Trans. Electron Devices 29, 64 (1982).

[53] A. Duwel, R. N. Candler, T. W. Kenny, and M. Varghese, Engineering MEMS resonators with low thermoelastic damping, J. Microelectromech. Syst. 15, 1437 (2006).

[54] J. J. Wortman and R. A. Evans, Young's modulus, shear modulus, and Poisson's ratio in silicon and germanium, J. Appl. Phys. 36, 153 (2004).

[55] G. A. Holzapfel, Nonlinear Solid Mechanics: A Continuum Approach for Engineering (Wiley, Chichester, 2002).

[56] P. Chadwick, Continuum Mechanics (Wiley, New York, 1976).

[57] P. R. Saulson, Thermal noise in mechanical experiments, Phys. Rev. D 42, 2437 (1990).

[58] J. M. L. Miller, A. Ansari, D. B. Heinz, Y. Chen, I. B. Flader, D. D. Shin, L. G. Villanueva, and T. W. Kenny, "Effective quality factor tuning mechanisms in micromechanical resonators" (to be published).

[59] Z. Mohammadi, J. M. L. Miller, K. L. Foster, T. W. Kenny, and L. G. Villanueva, "On resonator sensing improvement using linear feedback and parametric pumping" (to be published). 
[60] A. Eichler, J. Moser, J. Chaste, M. Zdrojek, I. WilsonRae, and A. Bachtold, Nonlinear damping in mechanical resonators made from carbon nanotubes and graphene, Nat. Nanotechnol. 6, 339 (2011).

[61] J. Atalaya, T. W. Kenny, M. L. Roukes, and M. I. Dykman, Nonlinear damping and dephasing in nanomechanical systems, Phys. Rev. B 94, 195440 (2016).

[62] B. Kim, M. A. Hopcroft, R. N. Candler, C. M. Jha, M. Agarwal, R. Melamud, S. A. Chandorkar, G. Yama, and
T. W. Kenny, Temperature dependence of quality factor in MEMS resonators, J. Microelectromech. Syst. 17, 755 (2008).

[63] J. Harley and T. W. Kenny, $1 / f$ noise considerations for the design and process optimization of piezoresistive cantilevers, J. Microelectromech. Syst. 9, 226 (2000).

[64] O. N. Tufte and E. L. Stelzer, Piezoresistive properties of heavily doped $n$-type silicon, Phys. Rev. 133, A1705 (1964). 